\title{
On the highest Lyubeznik number
}

\author{
BY KEN-I('HIROH KAWANAKI \\ Department of Hathematics. Nara Lnicersity of Eduration. Takabatake-cho. \\ Nara. 630-85:8. Japan. \\ ค-mail: kawaken@nara-edu.ac.jp
}

(Received 20 . Harch 2000 : rerised 27 s'eptember 2000 )

\begin{abstract}
In this paper: we will prove that the highest Lyubeznik number $\lambda_{d, d}(A)$ is one if $A$ is a cohen-Macaualy local ring containing a field. where $d$ is the dimension of $A$.
\end{abstract}

We assume that all rings are commutative and noetherian with identity throughout this paper.

\section{Introduction}

In this paper: we shall prove:

Theorex 1. Let $A$ be a local ring containing a field with dimension d. If $A$ is cohenMaraualy. then we hace $\lambda_{d, d}(A)=1$.

The investigation of the structure of local cohomology modules $H_{Y^{2}}^{2}(\tilde{\mathscr{F}})$ was initiated by Grothendieck and is a rery interesting subject in a field of commutative algebra. where $Y$ is a closed subscheme of a scheme $X$ and $\mathscr{F}$ is a quasi-coherent $\mathbb{C}_{X}$-module. Although several authors have developed very interesting results and deep theories (see for example, [F1]. [F2]. |G1|. |G2]. [Ha1|. [Ha3]. |Ha4]. [HaS]. $[\mathrm{HoR}]$. [HuK]. [HuL]. [O]. [PS]. [Sh]). these modules are still very mysterious. For finiteness property (for example. cofiniteness. finiteness of Bass numbers). several results are known (see. for example. [D]. [Yo]. [DM]. [Me]. see also, $[\mathbf{M o}]$. $[\mathbf{T}]$ and YYa| for results of local cohomology modules with supports in monomial ideals). In particular. Huneke and Sharp $[\mathbf{H u S}]$ and Lỵueznik $[\mathbf{L 1}]$ proved remarkable results and further. Lyubeznik defined a numerical invariant of local rings with respect to local cohomology modules [L1, theorem-definition $4 \cdot 1$ ]:

Definition 1. Let $A$ be a local ring of dimension $d$ whtch admits a surjective ring homomorphism $\pi: R \rightarrow A$, where $R$ is a regular local ring of dimension $n$ containing a field. Set $I=\operatorname{ker} \pi$ and let $m$ be the maximal ideal of $R$. Then the Bass number $\mu_{p}\left(\mathrm{~m}, H_{I}^{n-\imath}(R)\right)$ is finite and depends only on $A$. $i$ and $p$. but neither on $R$ nor on $\pi$. We denote this invariant by $\lambda_{p, l}(A)$. and we call this number the Lyubeznik number (or the $(p, i)$-Lyubeznik number). 
A complete local ring containing a field is always a surjective image of a regular local ring containing a field. so, if $A$ is a local ring containing a field. but not necessarily a surjective image of a regular local ring containing a field. one can set $\lambda_{p, z}(A)=\lambda_{p, z}\left(A^{\wedge}\right)$, where $A^{\wedge}$ is the completion of $A$ with respect to the maximal ideal.

We recall some basic properties of $\lambda_{p, 2}$ ((f. [L1. ( $\left.\left.4 \cdot 4 \mathrm{i}-\mathrm{r}\right)\right]$ ).

Theorem 2 (Lyubeznik). Let $A$ be a local ring of dimension $d$ containing a field. Then the following assertions hold:

(i) $\lambda_{p, l}(A)=0$ if $i>d$ :

(ii) $\lambda_{p, 2}(A)=0$ if $p>i$ :

(iii) $\lambda_{d, d}(A) \neq 0$ :

(iv) if $A$ is analytically normal. then $\lambda_{d, d}(A)=1$;

(v) if $A$ is a complete intersection. then $\lambda_{d, d}(A)=1$.

These results lead Lỵubeznik to ask the following question [L1. question 4.5].

Question 1 (Lycubeznik). Is it true that $\lambda_{d, d}(A)=1$ for all $A$ ?

Recently Walther answered this question negatively [W. proposition 3·2], using the Brodmann sequence. whose rings are not ('ohen-Macaulay. So we refine the above question as follows:

Question 2. Is it true that $\lambda_{d, d}(A)=1$ for ('ohen-Macaulay rings $A$ !

This is true for Cohen-Macaulay local rings $A$ of characteristic $p$ by the result of [PS. proposition $4 \cdot 1 \mid$, since the spectral sequence $H_{\mathrm{m}}^{p} H_{I}^{q}(R) \Rightarrow H_{\mathrm{m}}^{p+q}(R)$ degenerates. In this paper. we affirmatively answer Question 2. that is we will prove that if $A$ is a Cohen-Macaulay local ring containing a field of dimension $d$. then the highest Lyubeznik number $\lambda_{d, d}(A)$ is one.

This paper is dedicated to Profensor Yukitoshi Hinohara on the occasion of his seventieth birthday.

\section{Proof of the lemimas}

Definition 2. Let $A$ be a ring. For a positive integer $k$. we say that $A$ satisfies the Serre $\left(S_{k}\right)$-condition if it holds $\operatorname{depth}\left(A_{P}\right) \geqslant \inf (k, \operatorname{ht}(P))$ for all $P \in \operatorname{spec}(A)$ (cf. [Ma1. (17. 1). p. 125]).

Remark 1. If a catenary local ring $A$ satisfies the Serre $\left(S_{2}\right)$-condition, then $A$ is equidimensional, that is to say, all the irreducible components of $X=\operatorname{spec} A$ have the same dimension (ef. [Ha2. remark 2.4·1. p. 503]).

The following lemma is probably well known to experts.

Lenna 1. Let $\phi:(A, \mathrm{~m}) \rightarrow\left(A^{\prime}, \mathrm{nt}^{\prime}\right)$ be a local homomorphism of local rings $A . A^{\prime}$ with the maximal ideals m. m'. respectively. Suppose that the going-doun theorem holds for $\phi$ (see $\left[\right.$ Ma1. (5. A). p. 31] for the definition) and $\mathrm{m}^{\prime}=\mathrm{m} A$. If the dimension of $A$ is equal to $d$, then the dimension of $A^{\prime}$ is equal to $d$.

Proof. First note that if $I$ is an ideal of $A$, then the extension $\sqrt{I} A^{\prime}$ of the radical of $I$ is contained in the radical $\sqrt{I A^{\prime}}$ of the extension of $I$. Let $x_{1}, \ldots, x_{d}$ be a system of parameters of $A$. Then the radical $\sqrt{\left(x_{1}, \ldots, x_{d}\right)}$ is equal to $m$. It follows that $\sqrt{\left(x_{1}, \ldots, x_{d}\right) A^{\prime}} \supseteq \sqrt{\left(x_{1}, \ldots, x_{d}\right)} A^{\prime}=\mathrm{m} A^{\prime}=\mathrm{m}^{\prime}$. Since $\mathrm{m}^{\prime}$ is the maximal ideal of 
$A^{\prime}$, we have $\sqrt{\left(x_{1}, \ldots, x_{d}\right) A^{\prime}}=\mathrm{wt}^{\prime}$. Hence it holds that $\operatorname{dim} A^{\prime} \leqslant d=\operatorname{dim} A$. since the arithmetic rank of $m^{\prime}$ is not more than $d$. On the other hand. since $m$ is the maximal ideal. we have $\mathrm{m} A^{\prime} \cap A=\mathrm{m}$. Since the going-down theorem holds for $\phi$ and $m A^{\prime} \cap A=m$. we have the inequality htm $\leqslant$ htm' by the definition that the going-down theorem holds between $A$ and $A^{\prime}$ (cf. |Ma1. (5. A). p. 31|). Then it follows that $\operatorname{dim} A \leqslant \lim A^{\prime}$. We therefore conclucle that $\operatorname{dim} A=\operatorname{dim} A^{\prime}=d$.

We shall collect some results as lemmas for the proof of Proposition 1 and Theorem 1.

Lexus 2. Let $(A, \mathrm{~m})$ be a local ring with the maximal ideal m. Then the following assertions hold:

(i) $\left(A^{\wedge}, \mathrm{m} A^{\wedge}\right)$ is a local ring with the maximal ideal $m A^{\wedge}$ :

(ii) if $A$ is ('ohen-Macaulay (respectively regular). then $A^{\wedge}$ is ('ohen-Macaulay (respectively regular);

(iii) if $A$ is a homomorphic image of a l'ohen-Haraulay local ring and satisfies the serre $\left(S_{k}\right)$-rondition, then $A^{\wedge}$ satisfies the Serre $\left(S_{k}\right)$-rondition for a positive integer $k$ :

(iv) the natural map $A \rightarrow A^{\wedge}$ is a faithfully flat extension:

(v) if $A=R / I$ for some local ring $R$ and an ideal $I$ of $R$, then it holds that $A^{\wedge}=$ $R^{\wedge} / I R^{\wedge}$ :

(vi) if the dimension of $A$ is equal to $d$. then the dimension of $A^{\wedge}$ is equal to $d$. where $A^{\wedge}$ is the completion of $A$ with respect to the maximal ideal m.

Proof. Statement (i) follows from [Ma2, theorem. section 8 (4), p. 63] and statement (ii) follows from [Ma2. theorem 17.5 (ii). p. 136] and |Ma2. lines 13-14 in the proof of theorem 19.5 (ii). p. 157]. Further, statement (iii) follows from |Ma2. exercises to section 23. 23.2. p. 185]. (iv) follows from [Ma1. (4. A). corollary. p. 27] and $\lfloor$ Ma1. (23. L), (orollary 1. p. 170]. and (v) follows from $\mid$ Ma2, theorem $8 \cdot 11$. p. 61]. Finally since the natural malp $A \rightarrow A^{\wedge}$ is flat by (iv). the going-clown theorem holds between $A$ and $A^{\wedge}$ by [Ma1, (5. D). theorem 4. p. 33]. Therefore assertion (vi) follows from Lemma 1.

Lenna 3. Let $(A, \mathrm{nt})$ be a local ring with the maximal ideal m. Then the following assertions hold:

(i) $A^{\text {sh }}$ is a local ring with the maximal ideal $\mathrm{m} A^{\text {sh }}$ :

(ii) if $A$ is ('ohen-Maraulay (respectively regular). then $A^{\text {sh }}$ is ('ohen-Masaulay (respectively regular):

(iii) if A satisfies the serre $\left(S_{k}\right)$-condition, then $A^{\text {sh }}$ satisfies the serre $\left(S_{k}\right)$-condition for a posilice integer $k$ :

(iv) the natural map $A \rightarrow A^{\text {sh }}$ is a faithfully flat extension:

(v) if $A=R / I$ for some local ring $R$ and an ideal $I$ of $R$. then it holds that $A^{\text {sh }}=$ $R^{s h} / I R^{s h}$ :

(vi) if the dimension of $A$ is equal to $d$. then the dimension of $A^{\text {sh }}$ is equal to $d$. where $A^{\text {sh }}$ is a strict henselization of $A$ (see $[$ G3. (18-8). p. 144] for the definition).

Proof. Statements (i) and (iv) follow from [G3. proposition (18.8.8) (i), p. 146] and [G3. proposition (18.8.8) (iii). p. 147|. Statements (ii) and (iii) follow from $[$ G3. corollary $(18 \cdot 8 \cdot 13)(\mathrm{a}-\mathrm{c})$. p. 149]. and (v) follows from [Mil. p. 38. line 27|. Finally. 
since the natural map $A \rightarrow A^{\text {sh }}$ is flat by (iv). the going-down theorem holds between $A$ and $A^{\text {sh }}$ by [Ma1. (5. I)), theorem 4, p. 33]. Therefore assertion (vi) follows from (i) and Lemma 1.

Lexnat 4. Let $(A, \mathrm{~m})$ be a local ring with the maximal ideal $\mathrm{mt}$. Let $B$ be the ring $\left(\left(A^{\wedge}\right)^{s h}\right)^{\wedge}$. Then the following assertions. hold:

(i) $B$ is a loral ring with the maximal ideal $\mathrm{m} B$ :

(ii) if $A$ is Cohen-Hacaulay (respectirely regular), then $B$ is Cohen-1Macaulay (respectively regular):

(iii) if $A$ is a homomorphic image of a ('ohen-llacaulay local ring and satisfies the Serre $\left(S_{k}\right)$-condition. then $B$ satisfies the serre $\left(S_{k}\right)$-condition for a positive integer $k:$

(iv) the natural map $A \rightarrow B$ is a faithfully flat extension:

(v) if $A=R / I$ for some local ring $R$ and an ideal $I$ of $R$, then it holds that $B=$ $\left(\left(R^{\wedge}\right)^{s h}\right)^{\wedge} / I\left(\left(R^{\wedge}\right)^{s h}\right)^{\wedge}$ :

(ri) if the dimension of $A$ is equal to $d$. then the dimension of $B$ is equal to $d$.

where $A^{\wedge}$ is the completion of $A$ with respect to the maximal ideal, and $A^{\text {sh }}$ is a strict henselization of $A$.

Proof. We only prove statement (iii). Repeating a similar argument. the other assertions follow immediately from Lemmas 1-3. and |Ma1. (4. B). p. 27].

Let $R$ be a Cohen-Macaulay local ring which surjectively maps to a local ring $A$. We denote the kernel of the surjection by $I$. Then $A$ is isomorphic to $R / I$. If $A$ satisfies the Serre $\left(S_{k}\right)$-condition, then $A^{\wedge}$ satisfies the Serre $\left(S_{k}\right)$-condition by (iii) of Lemma 2 . since $A$ is a homomorphic image of a ('ohen-Nacaulay local ring $R$. Since $R$ is a ('ohen-Macaulay local ring. $R^{\wedge}$ is also a ('ohen-Maraulay local ring by (ii) of Lemma 2. Further $A^{\wedge}$ is isomorphic to $(R / I)^{\wedge}=R^{\wedge} / I R^{\wedge}$ by $(\mathrm{v})$ of Lemma 2 . Hence $A^{\wedge}$ is a homomorphic image of a C'ohen-Nacaulay local ring $R^{\wedge}$.

Since $A^{\wedge}$ satisfies the Serre $\left(S_{k}\right)$-condition. $\left(A^{\wedge}\right)^{\text {sh }}$ satisfies the Serre $\left(S_{k}\right)$-condition by (iii) of Lemma 3. Since $R^{\wedge}$ is a C'ohen-Macaulay local ring. $\left(R^{\wedge}\right)^{\text {sh }}$ is also a ('ohenMacaulay local ring by (ii) of Lemma 3. Further $\left(A^{\wedge}\right)^{\text {sh }}$ is isomorphic to $\left((R / I)^{\wedge}\right)^{\text {sh }}=$ $\left(R^{\wedge}\right)^{s h} / I\left(R^{\wedge}\right)^{s h}$ by $(\mathrm{v})$ of Lemma 3. Hence $\left(A^{\wedge}\right)^{s h}$ is a homomorphic image of a Cohen-Macaulay local ring $\left(R^{\wedge}\right)^{\text {sh }}$.

Now since $\left(A^{\wedge}\right)^{\text {sh }}$ is a homomorphic image of a ('ohen-Macaulay local ring $\left(R^{\wedge}\right)^{s h}$ and $\left(A^{\wedge}\right)^{s h}$ satisfies the Serre $\left(S_{k}\right)$-condition. $B=\left(\left(A^{\wedge}\right)^{s h}\right)^{\wedge}$ satisfies the Serre $\left(S_{k}\right)$ condition by (iii) of Lemma 2 . The proof of assertion (iii) is completed.

Lemna 5. Let $(R, \mathrm{~m}, k)$ be a regular local ring of dimension $n$ containing a field, and $I$ an ideal of $R$ of dimension $d>1$. If $R / I$ satisfies the serre $\left(S_{2}\right)$-condition, then the following assertions hold:

(i) inj. $\operatorname{dim}_{R} H_{I}^{n-d}(R)=d$ :

(ii) inj. $\operatorname{dim}_{R} H_{I}^{J}(R)<n-1-j$ if $j>n-d$, where inj. $\operatorname{dim}_{R} T$ is the injectice dimension of an $R$-module $T$.

Proof. Statement (i) is straightforward from [L1. (4-4iii)]. so we only have to prove the assertion (ii). We proceed in several steps.

Step 1. First we prove that the 'Second Vanishing Theorem holds for the local cohomology module $H_{I}^{J}(R)$, that is $H_{I}^{J}(R)=0$ for $j \geqslant \operatorname{dim} R-1$ (cf. [Sp. p. 143, line 15]). 
Nince $A=R / I$ is a homomorphic image of regular local ring $R, B=\left(\left(A^{\wedge}\right)^{\text {sh }}\right)^{\wedge}=$ $\left(\left(R^{\wedge}\right)^{s h}\right)^{\wedge} / I\left(\left(R^{\wedge}\right)^{\text {sh }}\right)^{\wedge}$ is also a homomorphic image of regular local ring $\left(\left(R^{\wedge}\right)^{s h}\right)^{\wedge}$ by (ii) of Lemma 4 . If $A=R / I$ satisfies the Serre $\left(S_{2}\right)$-condition. then the local ring $B=\left(\left(A^{\wedge}\right)^{s h}\right)^{\wedge}=\left(\left(R^{\wedge}\right)^{s h}\right)^{\wedge} / I\left(\left(R^{\wedge}\right)^{s h}\right)^{\wedge}$ also satisfies the serre $\left(S_{2}\right)$-condition by (iii) of Lemma 4 , and the dimension of $B$ and $\left(\left(R^{\wedge}\right)^{\text {sh }}\right)^{\wedge}$ are equal to $d$ and $n$ respectively by (ri) of Lemma 4. It follows from $\mid \mathrm{Ha2}$. corollary 2.4. p. 5033 that the punctured spectrum of spec $(B)$ is comnected. that is spec $A$ is formally geometrically connected (see $[\mathrm{HuL}$. theorem $2 \cdot 9$. 1. 79$]$ for the definition). Hence since the dimension of $R / I$ is greater than one. we have $H_{I}^{n}(R)=H_{I}^{n-1}(R)=0$ by $[$ HuL. theorem 2.9. p. 79].

Step 2. Next we shall prove that $H_{I}^{J}(R)_{Q}=0$ for all prime ideals $Q$ containing $I$ with $\operatorname{dim} R / Q \geqslant n-1-j$ and $\operatorname{dim} R / Q<d-1$.

Incleed. let $Q$ be any prime ideal of $R$ containing $I$ with $\operatorname{dim} R / Q \geqslant n-1-j$ and $\operatorname{dim} R / Q<d-1$.

(a) If $\lim R / Q \geqslant n-1-j$, then we have:

$$
\begin{aligned}
j & \geqslant n-1-\operatorname{dim} R / Q \\
& =\lim R-1-\operatorname{dim} R / Q \\
& =(\operatorname{dim} R-\operatorname{dim} R / Q)-1 \\
& =\operatorname{lit} Q-1 \\
& =\lim R_{Q}-1,
\end{aligned}
$$

by [Ma2. section 31. lemma 2. p. :200|.

(b) Further since $R / I$ satisfies the Serre $\left(S_{2}\right)$-condition. $R / I$ is equidimensional by Remark 1. Hence it holds that

$$
\begin{aligned}
\operatorname{dim}(R / I)_{Q} & =\operatorname{ht} Q / I \\
& =\operatorname{dim} R / I-\operatorname{dim}((R / I) /(Q / I)),
\end{aligned}
$$

by [Ma2, section 31. lemma 2. p. 250) again. If $\operatorname{dim} R / Q<d-1$. then we have:

$$
\begin{aligned}
\operatorname{dim} R_{Q} / I R_{Q} & =\operatorname{dim}(R / I)_{Q} \\
& =\mathrm{ht} Q / I \\
& =\operatorname{dim} R / I-\operatorname{dim}((R / I) /(Q / I)) \\
& =\operatorname{dim} R / I-\operatorname{dim} R / Q \\
& >d-(d-1) \\
& =1,
\end{aligned}
$$

by |Ma2. section 31. lemma 2. p. 250|.

We note that the localization $(R / I)_{Q}=R_{Q} / I R_{Q}$ satisfies the Serre $\left(S_{.2}\right)$-condition and $R_{Q}$ is also a regular local ring.

Now we consider the category of modules over the regular local ring $R_{Q}$. It then follows from Step 1 that the Second Vanishing Theorem holds for the local cohomology module $H_{I R_{Q}}^{J}\left(R_{Q}\right)$. that is. $H_{I}^{J}(R)_{Q}=0$ for $j \geqslant \operatorname{dim} R_{Q}-1$ and $\operatorname{dim} R_{Q} / I R_{Q}>1$ by $(a)$ and $(b)$. We obtain the assertion of this step.

Step 3. In this step. we shall prove that $\operatorname{dim} \operatorname{Supp}_{l}^{J}(R)_{Q}<n-1-j$ under the assumption that $j>n-d$.

Indeed. let $Q$ be in Supp $H_{I}^{J}(R)$. then we have $H_{I}^{J}(R)_{Q}=H_{I R_{\Omega}}^{J}\left(R_{Q}\right) \neq 0$. From the 
assumption $j>n-d$, we have

$$
\begin{aligned}
\operatorname{dim} R / Q & \leqslant n-1-j \\
& <n-1-(n-d) \\
& =d-1 .
\end{aligned}
$$

It follows from Step $2(b)$ that $\operatorname{dim} R_{Q} / I R_{Q}>1$. Then it holds that

$$
\begin{aligned}
3 & \leqslant \lim R_{Q}-1 \\
& =\operatorname{dim} R-\operatorname{dim} R / Q-1 \\
& =n-1-\operatorname{dim} R / Q .
\end{aligned}
$$

by the local Lichtenbaum-Hartshorne Vanishing Theorem and the (irothendieck Vanishing Theorem for the local cohomology module $H_{I}^{J}(R)_{Q}=H_{I R_{Q}}^{J}\left(R_{Q}\right) \neq 0$. It therefore follows from Ntep 2 that $\operatorname{dim} R / Q<n-1-j$ for all prime ideals $Q$ in $\operatorname{Supp} H_{I}^{\jmath}(R)$. We then conclude that $\operatorname{dim} \operatorname{supp} H_{I}^{J}(R)<n-j-1$.

Step 4. Since the inequality inj.dim $H_{I}^{J}(R) \leqslant \operatorname{dim} \operatorname{supp}_{I}^{J}(R)$ holds by $[\mathbf{H u S}]$ and [L1. corollary 3.6. p. 52], the proof of the assertion (ii) is completed.

\section{Proof of the main results}

PRoposition 1. Let $R$ be a regular local ring containing a field of dimension $n$, $I$ an ideal of $R$ of dimension $d>1$. If $R / I$ satisfies the Serre $\left(S_{2}\right)$-condition. then we have $\lambda_{d . d}(R / I)=1$.

Proof. The functor $\Gamma_{I}(-)$ takes injectives into $\Gamma_{m}(-)$-acrelic objects in the category of $R$-modules. So by (irothendieck's spectral sequence. we obtain the following spectral sequence (cf. [G1, theorem A, p. 5]):

$$
E_{\stackrel{2}{p, q}}^{p,} H_{\mathrm{m}}^{p} H_{I}^{q}(R) \Longrightarrow H^{p+q}=H_{\mathrm{m}}^{p+q}(R) .
$$

The spectral sequence has the differentials as follows:

$$
E_{r}^{d-r, n-d-(1-r)} \longrightarrow E_{r}^{d, n-d} \longrightarrow E_{r}^{d+r, n-d+(1-r)} .
$$

We shall prove that all the differentials that come into and go out of $E_{r}^{d, n-d}$ are 0 for all $r \geqslant 2$.

Now it follows from [L1. (4·+i), p. 54] that $H_{m}^{p} H_{I}^{n-\imath}(R)=0$ for all $i>d$ and all $p \geqslant 0$. On the other hand. we calculate as follows:

$$
\begin{aligned}
-(-d+(1-r)) & =d-1+r \\
& \geqslant d-1+2 \\
& =d+1 \\
& >d,
\end{aligned}
$$

for $r \geqslant 2$. Hence we have $E_{2}^{d+r, n-d+(1-r)}=H_{m}^{d+r} H_{I}^{n-d+(1-r)}(R)=0$. So it holds that $E_{r}^{d+r, n-d+(1-r)}=0$ for all $r \geqslant 2$. Thus it follows that the differentials $E_{r}^{d, n-d} \rightarrow$ $E_{r}^{d+r, n-d+(1-r)}$ are 0 for all $r \geqslant 2$.

From Lemma 5, we calculate as follows:

$$
\begin{aligned}
\operatorname{inj} . \operatorname{dim} H_{I}^{n-d-(1-r)}(R) & <n-1-(n-d-(1-r)) \\
& =d-r .
\end{aligned}
$$

Hence we have $E_{2}^{d-r, n-d-(1-r)}=H_{\mathrm{m}}^{d-r} H_{I}^{n-d-(1-r)}(R)=0$. So it holds that 
$E_{r}^{d-r . n-d-(1-r)}=0$ for all $r \geqslant 2$. Thus it follows that the differentials $E_{r}^{d-r, n-d-(1-r)} \rightarrow$ $E_{r}^{d, n-d}$ are 0 for all $r \geqslant 2$.

Further we have $E_{2}^{p, q}=0$ for all $p \geqslant 0, q \geqslant 0$ with $p+q=n, p<d$ by Lemma 5 . Since inj.dim $H_{I}^{q}(R) \leqslant \operatorname{dim} \operatorname{supp}_{I}^{q}(R) \leqslant \operatorname{dim} V(I)=d$ by [HuS] and [L1. corollary 3.6. p. 52]. it holds that $E_{2}^{p, q}=0$ for all $p \geqslant 0 . q \geqslant 0$ with $p+q=n . p>d$. It then follows that $E_{22}$-terms are all 0 in the total degree $n$ except $E_{2}^{d, n-d}$.

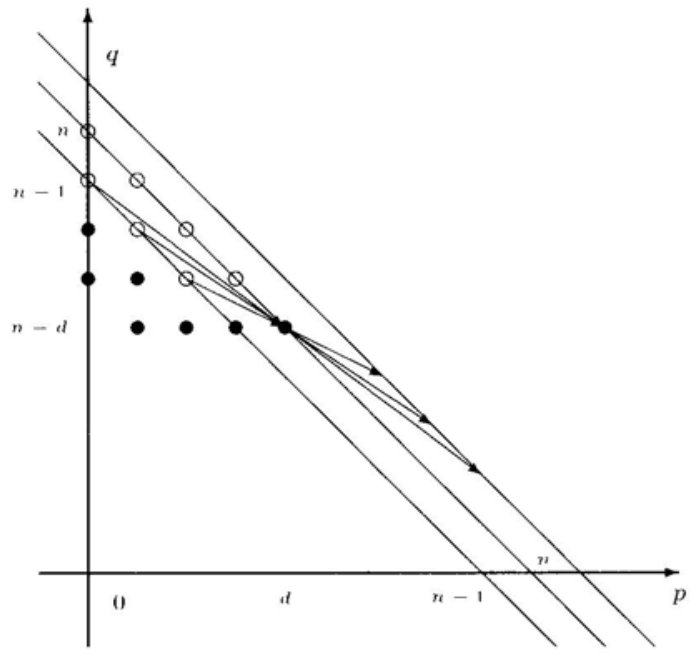

Fig. 1

We express $E_{2}$-terms in the above diagram (Fig. 1). The circles mean the vanishing of $E_{2}$-terms by Lemma 5 . Furthermore all $E_{2}$-terms are 0 except the black circles.

Therefore the above spectral sequence collapses at $E_{2}^{d, n-d}$ and we have isomorphisms:

$$
\begin{aligned}
H_{\mathrm{m}}^{d} H_{I}^{n-d}(R) & =E_{2}^{d . n-d} \\
& \simeq E_{\times}^{d . n-d} \\
& \simeq H^{n} \\
& =H_{\mathrm{m}}^{n}(R) .
\end{aligned}
$$

Since $R$ is a regular local ring. $H_{m 1}^{n}(R)$ is isomorphic to $E(k)$. where $E(k)$ is the injective hull of $k$. Since $H_{m}^{d} H_{I}^{n-d}(R)$ is isomorphic to $E(k)$. it therefore follows from $[\mathbf{L} 1$. lemma 1.4, p. 4t] that $\lambda_{d . d}(A)=1$. The proof of the proposition is completed.

Proof of Theorem 1. Completing the local ring $A$ with respect to the topology defined by the maximal irleal. there is a surjection $R \rightarrow A^{\wedge}$ from a regular local ring $R$ containing a fields to $A^{\wedge}$ by ('ohen's structure theorem. We denote its kernel by $I$ and the maximal ideal of $R$ by $m . A^{\wedge}=R / I$ is also ('ohen Macaulay by (i) of Lemma 3. Especially $A^{\wedge}=R / I$ satisfies the Serre $\left(S_{.2}\right)$-condition by $\lceil\mathbf{M a 1}$. (17. I). p. 125. lines 8-9|. Therefore the theorem follows from Proposition 1.

('OROLlaRs 1. Let $A$ be a local ring containing a field of dimension $d>1$, complete with the topology defined by the maximal ideal. If $A$ satisfies the serre $\left(S_{2}\right)$-condition. then we have $\lambda_{d, d}(A)=1$.

Proof. By C'ohen's structure theorem. there is a surjection from a regular local ring $R$ to $\dot{A}$. Therefore the assertion follows from Proposition 1. 
Remark 2. If the dimension of a ring $A$ is equal to one, then it always holds that $\lambda_{1,1}(A)=1$.

Example 1. The converse of the theorem, the proposition and the corollary do not hold in general. The following example is essentially due to Kazufumi Eto. Let $R$ be the localization of $k\left[x_{1}, x_{2}, x_{3}, x_{4}, x_{5}, x_{65}\right]$ by $\left(x_{1}, x_{2}, x_{3}, x_{4}, x_{5}, x_{6}\right), I=\left(x_{1}, x_{2}, x_{3}\right) \cap$ $\left(x_{2}, x_{3}, x_{4}\right) \cap\left(x_{3}, x_{4}, x_{5}\right) \cap\left(x_{4}, x_{5}, x_{65}\right) \cap\left(x_{5}, x_{6}, x_{1}\right)$ and $P=\left(x_{1}, x_{2}, x_{3}, x_{5}, x_{6}\right)$. Here we note that $I$ is equidimensional and $\operatorname{dim} R / I=3$. Then we have $\operatorname{dim}(R / I)_{P}=2$ and $I R_{P}=\left(x_{1}, x_{2}, x_{3}\right) R_{P} \cap\left(x_{5}, x_{6}, x_{1}\right) R_{P}$. It follows that the punctured spectrum of Spec $(R / I)_{P}$ is disconnected. that is $\mathrm{Spec} R / I$ is not locally connected in eodimension one (see $[\mathrm{Ha} 2$, definition, p. $5(0)]$ for the definition). It follows from $[\mathrm{Ha2}$, corollary 2.4. p. 503] that Spec $R / I$ does not satisfy the $\left(S_{2}\right)$-condition and therefore $R / I$ is not ('ohen-Macaulay:

On the other hand. Yanagawa shows that for pure square-free monomial ideals, the highest Lyubeznik number is one if and only if the corresponding ring is connected in codimension one (cf. [Ya. corollary 3.16]). The local space spec $R / I$ is connected in codimension one and $I$ is a pure square-free monomial ideal in $R$. It follows from [Ya, corollary $3 \cdot 16$ ] that $\lambda_{3.3}(R / I)=1$. While he used the combinatorial argument to prove [Ya. corollary 3.16]. one can find the direct proof of the result that $\lambda_{3,3}(R / I)=$ $1 \mathrm{in}$ [EK], by which we could point out that the earlier version of [Ya, corollary $3 \cdot 16$ ] was incorrect.

Acknowledgements. The author is grateful to Professor Yukitoshi Hinohara for his successive encouragement during work on this paper. He also thanks Professors Mitsuhiro Miyazaki and Kiriko Kato for valuable comments. Special thanks are due to the referee for reading carefully the earlier version of this paper.

\section{REFEREN('ES'}

[AM] II F. ATISAH and I. G. M.A'DONAL1). Introduction to commutative algebra. Addison-Wesley Series in Math. (Reading. Massachusetts, 1969).

[D D. Detrixo. On cotiniteness of local cohomology modules. Math. Proc. ('amb. Phil. Soc. 115 (1994). $421-429$.

|DM| D. Delfixo and T. Marlex. ('ofinite modules and local cohomology. J. Pure Appl. Algebra $121(1997) \quad 45-52$.

[EK K. ETo and K -I. KAWAstKI. In preparation.

[F1] (:. Faltixas. Cher die Annulatoren lokaler Kohomologiegruppen. Archir der Math. 30 (1978). $473-476$.

[F2| (4. Falisix.s. Cher lokale Kohomologiegruplen hoher (Ordnung. J. Reine Angew. Math. $313(1980)+3-.51$.

[G1] A. Grothexines. Loral cohomolog!y. notes by Hartshorne Lect Notes Math. vol. 41 (Springer-Verlag, 1967).

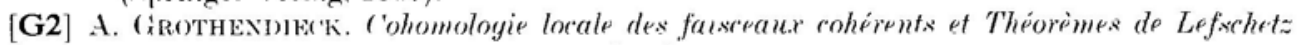
locaux et globaux (SGG.A) (North-Holland. 1968).

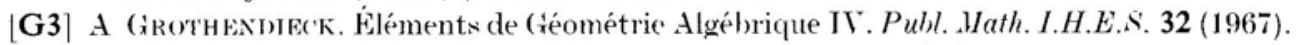

[Ha1] R. Hartshorse. Affine duality and cofiniteness. Iment Hath 9 (1970), 145-164.

[Ha2] R. HARTshorsk. ('omplete intersections and connecterlness. Amer J. Math. 84 (1962). $497-508$.

[Ha3] R. Haktshorne. C'ohomological dimensıon of algebraic vartetıes. Ann. Math. 88 (1968). $403-450$.

[Ha4] R. Hartshorix. Lectures on the Girothendieck Inality theory. Lect. Notes Math vol. 20 (Springer-Verlag. 1966).

[HoR $\mid$ M. Hocheter and J. RoberTs. The purity of the Frobenius and Local cohomology. Adr: Iath. 21 (2) (1976). 117-172 
[HaO] $R$ HART'HORNE and A. Ocil's On the tactoriality of local rings of small embedding colimension. ('omm. in Aly. 1 (5) (1974). 415-437.

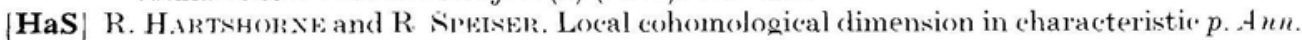
Wath. 105 (1977) . 4.) 79

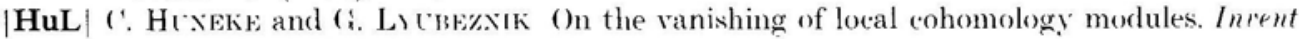
Hath. $102(199(0) .73-993$

[HuK] ('. HINEkE and J. KoH ('ofiniteness and ranshing of local cohomology modules . Wath Proc. ('amb. Phil. Sor. 110 (1991). 421-429.

|HuS| ('. Hixeke and R. Y. NHARP. Bass numbers of local cohomology modules Trans. Amer. Iath. Soc. 339 (1993). 765-779

[L1 (i. Lribrasik. Finiteness properties of local cohomology modules (an application of $D$. modules to commutative algebra) Inrent. Wath. 102 (1993). 41-5̃).

LL2 (;. Lribexik F-modules applications to local cohomology and $D$-modules in chararteriste $p>$ 0. .J. Reine Angere. Hath. 491 (1997). 65-130

[Ma1] H. MATs's['RA. ('ommutatice algebra (Benjamin/('ummings 2nd ed.. 1980),

[Ma2] H Matsinete. ('ommutatue ring theory. ('ambridge studies in Advance Nathematicis. 8 (Cambridge L'niversity Press. 1986).

[Me] L. Melkersion Properties of eofinite modules and applications to local cohomology. Math. Proc. ('amb. Phil. Nox'. 110 (1999). 4:21-429.

[Mil .J. N. Mulxe. Étale ('ohomolog! (Princeton [niversity Press. 1966).

[Mo].J A. Moxtaxkr. ('haracteristic cyeles of local cohomology modules of monomial ideals. preprint.

|O A. Ocis. Local cohomological dimension of algebraic varieties. Ann. Math. 98 (1973), $1-34$.

[PS] ("PkskIxE and L. SzPro. Jnmension projective finie et cohomologie locale Publ Math I.H.E.S' 42 (1973) 32:3-395.

[Sh] R. Y. Sнавp The Frobenius homomorphism and local cohomology in regular local ring of positive eharacteristic J. Pure Appl. Algebra 71 (2-3) (1970), 313-317.

[Sp] R. Siptiskr. ('ohomological dimension of non-complete hypersurfaces Inient. Wath 21 (1973). 14:3-150.

[T] N. Terat. Local cohomology modules with respect to monomial ideals preprint.

[W C. Warther. Some calculations on the Lyubeznik numbers of a local ring. preprint.

[Yo] K.-1 Yoshme ('ofiniteness of local cohomology modules for dimension one ideals. Nagoya J. Math. 147 (1997). 179-191.

[Ya] K. Yaxtiall Bass numbers of local cohomologr modules w ith supports in monomial ideal. Wath. Proc. ('amb. Phil. Sor. 131 (2001), 45-60. 